\title{
Patient Model and One New Possibility Evaluation of Anaerobic Test
}

\author{
Milan Stork \\ Department of Applied Electronics and \\ Telecommunications/RICE \\ University of West Bohemia \\ Plzen, Czech Republic \\ stork@kae.zcu.cz
}

\author{
Jaroslav Novak \\ Department of Sports Medicine \\ Medical Faculty in Plzen \\ Charles University in Prague, \\ Plzen, Czech Republic \\ novakj@lfp.cuni.cz
}

\begin{abstract}
The Wingate Anaerobic test (WAT) is the most popular test for estimation a person's anaerobic capacity. Because anaerobic performance is important for many athletes, the most well-known application of the Wingate test is in sports medicine. But there is also more. The Wingate test is especially useful for children with chronic diseases like Cystic Fibrosis, Diabetes and Muscle Dystrophy. Another application is to test the increasing obese population. The test is based on cycling at maximal intensity, for 30 seconds, against a high braking force. This force remains constant throughout the test but, because it is so high, the subject cannot maintain the initial velocity for more than a few seconds, before starting to slow down. Some new devices enable fast sampling of load and other measured data and therefore is possible gain more information from test. In this paper the mathematical models based on data gained from WAT are described. The linear models based on system identification are used.
\end{abstract}

Keywords - Anaerobic, ergometer, heart rate, model, power, wingate

\section{INTRODUCTION}

The most commonly employed protocol for the measurement of anaerobic response is the 30-s WAT. Developed almost 30 years ago, the WAT involves a maximal exertion load on a cycle ergometer to evaluate peak power, mean power, and fatigue index; [1], [2]. When performing a $30 \mathrm{sec}$. WAT, a subject typically exhibits a sharp rise in power output, reaching peak power within the first few seconds. The ability of a person to perform physical work depends on skill, or muscle strength, speed of movement, or one's stamina. A common denominator of all these activities is that they are performed through muscle contraction. Such contraction can be performed only when biochemical energy is converted into mechanical energy. Two types of biochemical energy are distinguished [1-5]:

a) aerobic: energy is supplied to the muscles in the presence of oxygen

Milan Stork's participation was supported by Department of Applied Electronics and Telecommunications, University of West Bohemia, Plzen, Czech Republic and by the Ministry of Education, Youth and Sports of the Czech Republic under the RICE - New Technologies and Concepts for Smart Industrial Systems, project No. LO1607 and the Internal Grant Agency of University of West Bohemia in Plzen, the project SGS-2018-001 b) anaerobic: energy is supplied to the muscles in the absence of oxygen

Aerobic activities are typically of a low-tomoderate intensity and can be continued over minutes or even hours. Anaerobic activities are very intense and for that reason can only be continued for one minute or less. While aerobic fitness is a characteristic of the person as a whole, anaerobic fitness is a local characteristic of the muscle because of its independence on blood and oxygen supply to the muscle. This means that a person may have a high anaerobic performance in one muscle group and a lower anaerobic performance in another. The WAT is used world-wide and is considered the most popular test of anaerobic muscle performance [6-10]. The test is based on cycling at maximal speed, for 30 seconds, against a high braking force. This force remains constant throughout the test but, because it is so high, the subject cannot maintain the initial velocity for more than a few seconds and after power go slow down. The example of bicycle for WAT with control panel is shown in Fig. 1. During the test the following parameters are measured: Time [s], distance $\mathrm{km}$ ], power $[\mathrm{W}]$, heart rate (HR) [beats/min], cadence [rotation $/ \mathrm{min}]$, speed $[\mathrm{km} / \mathrm{h}]$. These parameters are sampled by sampling period $T_{\mathrm{S}}=0.125[\mathrm{~s}]$, therefore for 30 [s] test, the 240 samples are stored in memory and can be read after.

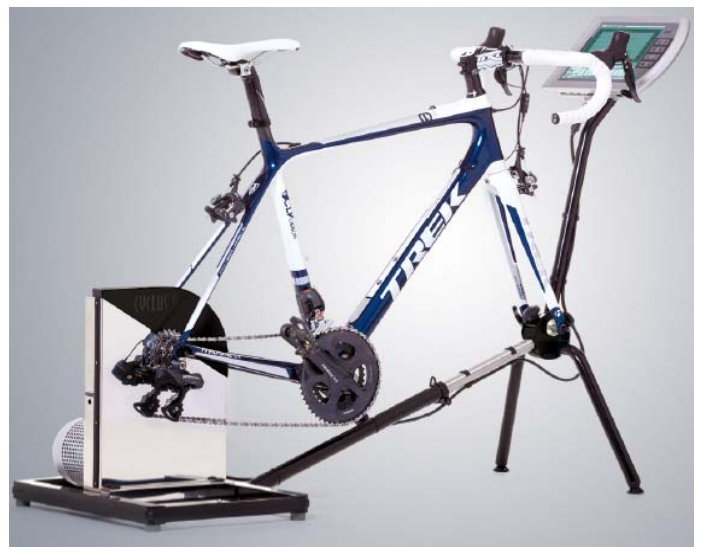

Figure 1. Example of ergometric bicycl for WAT test with control panel placed ahead from handlebars

The sampled data (with sampling period $0.125 \mathrm{~s}$ ) can be read as *.xls or *.csv files. 


\section{NEW WAT EVALUATION IN 3D}

The sampled data can be used for other types of test evaluation, e.g. 3 dimensional graph (3D) or graph containing curves of 2 or more subjects for comparison or comparison of 2 or more examination of one person. The example of examination displayed in 3D graph is shown in Fig. 2. Comparison of examination of 2 subjects in 3D is displayed in Fig. 3. persons by means of polynomial functions are shown.

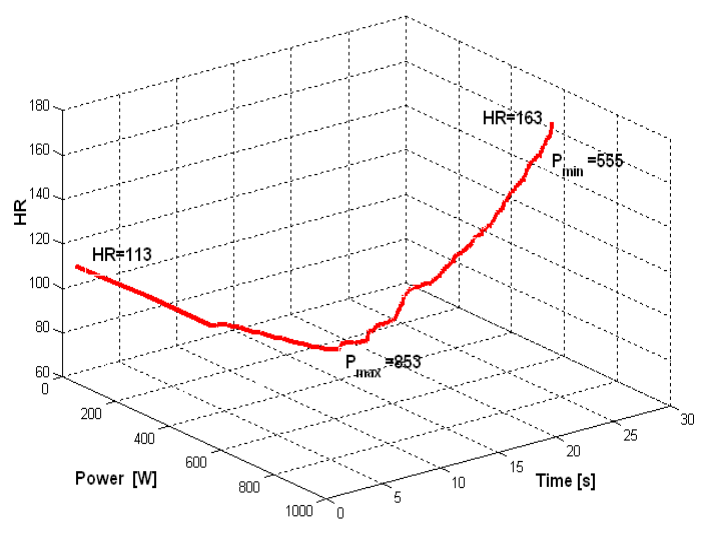

Figure 2. Example of one subject of WAT result displayed in 3 D graph. $\mathrm{X}$-axis time, $\mathrm{Y}$-axis load, Z-axis heart rate

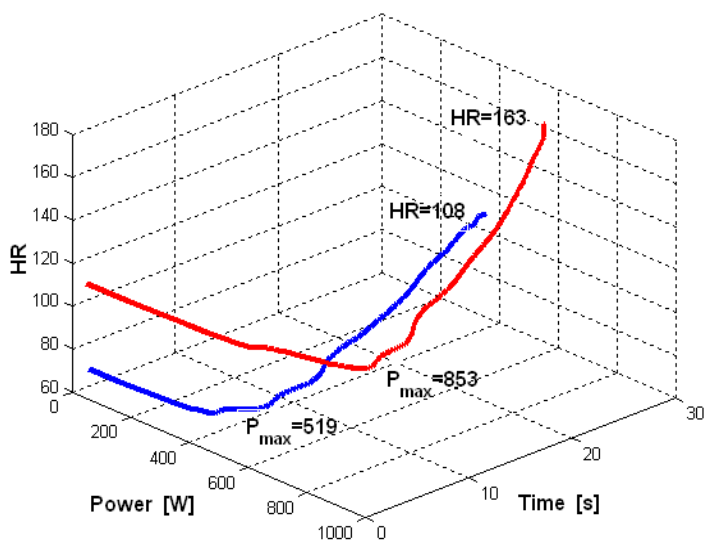

Figure 3. Example of WAT results of 2 subject displayed in $3 \mathrm{D}$ graph. $\mathrm{X}$-axis time, $\mathrm{Y}$-axis load, $\mathrm{Z}$-axis heart rate

\section{LINEAR MATHEMATICAL MODELS}

For some medical purposes the mathematical models of WAT is important. The mathematical models can be divided in linear and nonlinear. In this part the dynamical models of $H R$ versus power are presented. Example of time diagram power and $H R$ is shown in Fig. 4. This time evolution can be divided in 2 parts - first, from beginning to first maximal power peak (very short, usually up to several second, derivation of power $=0$ ) and second part, from first maximal power to the end. In Fig. 5, only the second part is displayed. From this Fig. 5 can be seen time delay in $H R$ as the response on load. Moreover, for linear mathematical model estimation the initial=resting heart rate (minimal value) is subtracted from all $H R$ samples and new vector of samples is marked as HR1. Results of different models (including nonlinear model) are shown in Fig. 6 with fit values.

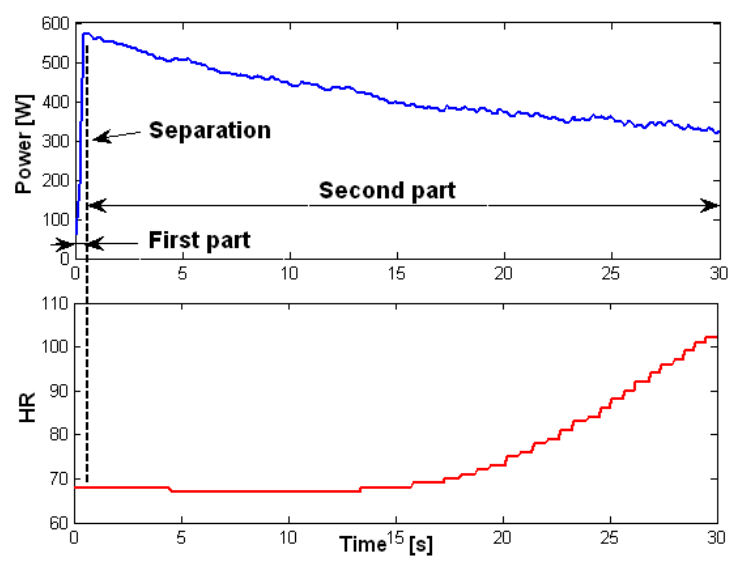

Figure 4. Exapmle of time evolution of power (top) and $H R$ (bottom), separated on 2 parts - first, from the beginnong to first maximal power peak and second, from the first maximal power peak to the end of test
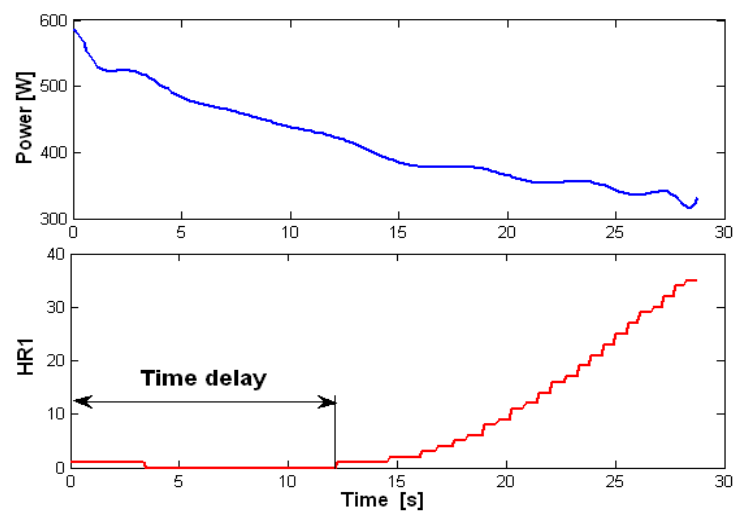

Figure 5. Only the second part of the test (from maximal power to the end). In $H R l$ - initial value of $H R$ (minimal value of $H R$ ) is subtracted from $H R$ and time delay of $H R$ is displayed

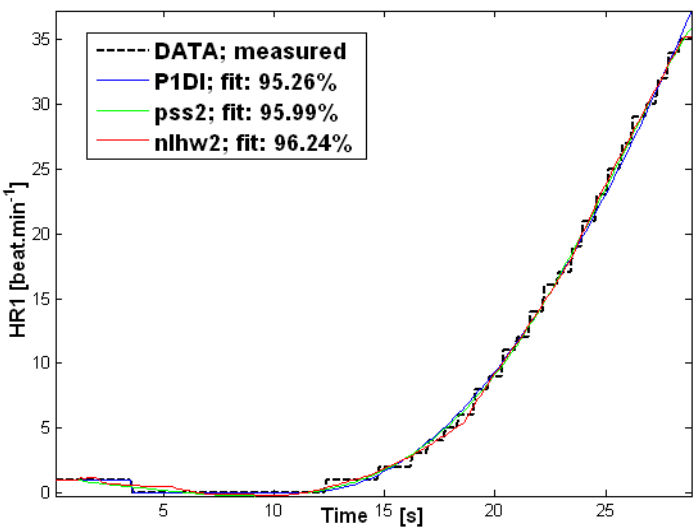

Figure 6. Results of different dynamical models used for approximation of WTAEquations

Several models were used for estimation of the WAT, see results in Fig. 9. P1D1 - linear; second order system consists of first order system and delay, pss2 - linear; second order state space system, nlhw2 - nonlinear; Hammerstein-Wiener model [11-13] (most complex). All presented models bring almost similar "fit" values, but linear models are simplest with good "fit" result. The equations of different types and orders of linear models with transfer functions are given by eq. (1)-(5). 
P1D - first order process model with time delay.

$$
\frac{H R l(s)}{\operatorname{pow}(s)}=\frac{y(s)}{u(s)}=\frac{K_{P}}{s\left(s T_{P 1}+1\right)} e^{-T_{D} s}
$$

P2D - second order process model with time delay (all poles are real).

$$
\frac{\operatorname{HRl}(s)}{\operatorname{pow}(s)}=\frac{y(s)}{u(s)}=\frac{K_{P}}{\left(s T_{P 1}+1\right)\left(s T_{P 2}+1\right)} e^{-T_{D} s}
$$

P2DU - second order process model with time delay. The letter $U$ is included to mark that underdamped modes (complex-valued poles) are permitted. If $\mathrm{U}$ is not included, all poles are restricted to be real.

$$
\frac{\operatorname{HRI}(s)}{\operatorname{pow}(s)}=\frac{y(s)}{u(s)}=\frac{K_{P}}{s^{2} T_{1}^{2}+2 \xi s T_{1}+1} e^{-T_{D} s}
$$

P3D - third order process model with time delay (all poles are real).

$$
\begin{aligned}
& \frac{\operatorname{HRl}(s)}{\operatorname{pow}(s)}=\frac{y(s)}{u(s)} \\
& =\frac{K_{P}}{\left(s T_{P 1}+1\right)\left(s T_{P 2}+1\right)\left(s T_{P 3}+1\right)} e^{-T_{D} s}
\end{aligned}
$$

P3DU - third order process model with time delay. Complex-valued poles are permitted

$$
\begin{aligned}
& \frac{\operatorname{HRI}(s)}{\operatorname{pow}(s)}=\frac{y(s)}{u(s)} \\
& =\frac{K_{P}}{\left(s^{2} T_{1}^{2}+2 \xi s T_{1}+1\right)\left(s T_{2}+1\right)} e^{-T_{D} s}
\end{aligned}
$$

where output $y(s)=H R l(s)$ is heart rate output without offset (initial $H R$ is subtracted from measuring values) and $u(s)=p o w(s)$ is input $=$ power.

In the next part the models estimation for 3 subject are presented.

Example 1- male, well trained subject.

\section{$\mathrm{P} 2 \mathrm{D}$}

$K p=10048 ; T_{p l}=2.16 \cdot 10^{6} ; T_{p 2}=8.7 ; T_{d}=0.47$

P3D

$K_{p}=79821 ; T_{p 1}=2.15 \cdot 10^{7} ; T_{p 2}=2.5 ; \quad T_{p 3}=2.53 ;$ $T_{d}=0.215$

P3DU

$K_{p}=1.001 \cdot 10^{5} ; \quad T_{1}=0.018 ; \quad \xi=241 ; T_{2}=2.15 \cdot 10^{7} ;$ $T_{d}=0.47$ Results are shown in Fig. 7, 8 and 9.

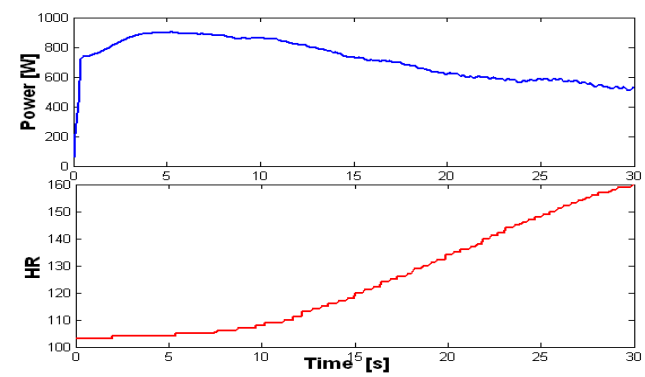

Figure 7. Exapmle 1. Time evolution of power (top) and $H R$ (bottom)

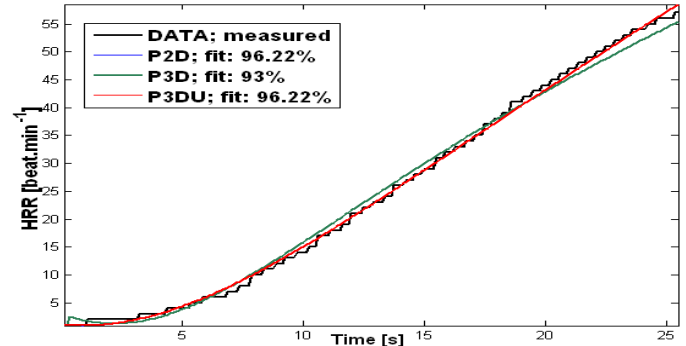

Figure 8. Exapmle 1. Results of different dynamical models used for estimation of WAT equations

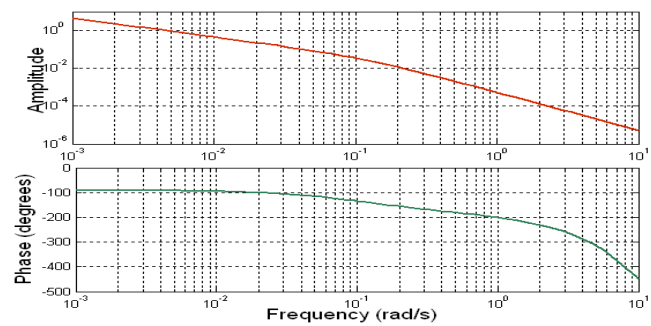

Figure 9. Exapmle 1. Bode diagram (P2D only)

Example 2 - female, well trained subject.

P3D

$K_{p}=2.75 \cdot 10^{6} ; T_{p l}=8530 ; T_{p 2}=8525 ; T_{p 3}=1014 ;$ $T_{d}=0$ Results are shown in Fig. 10, 11 and 12.

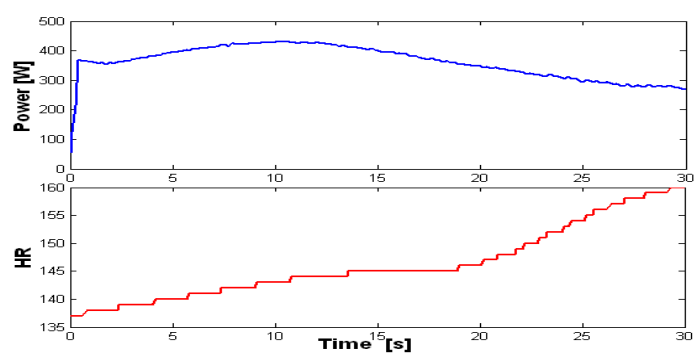

Figure 10. Exapmle 2. Time evolution of power (top) and $H R$ (bottom)

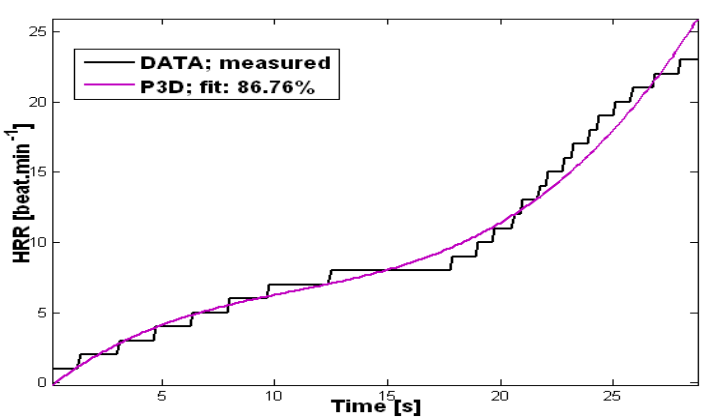

Figure 11. Exapmle 2. Results of dynamical model used for estimation of WAT equations

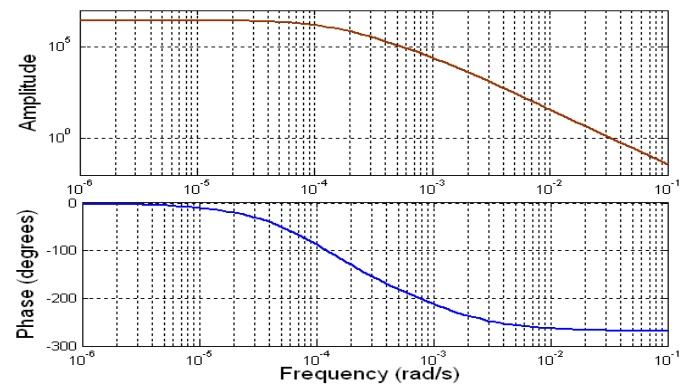

Figure 12. Exapmle 2. Bode diagram (P3D) 
Example 3 - male, well trained subject.

\section{P2D}

$K_{p}=6.94 \cdot 10^{7} ; T_{p I}=1.28 \cdot 10^{10} ; T_{p 2}=18.8 ; T_{d}=3.24$

P3D

$K_{p}=0.0234 ; \quad T_{p 1}=33.6 ; \quad T_{p 2}=17.5 ; \quad T_{p 3}=150 ;$

$T_{d}=2.73$ Results are shown in Fig. 13, 14 and 15 .

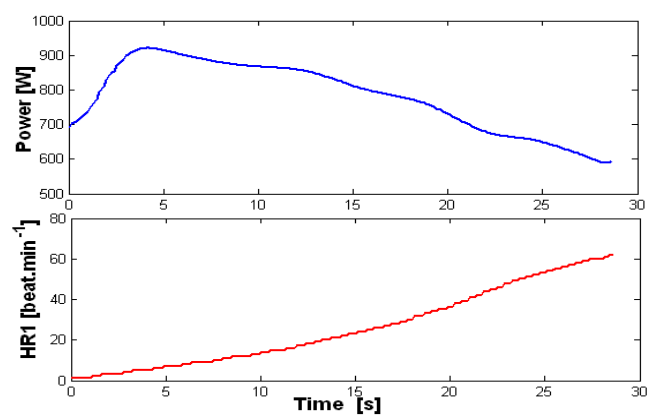

Figure 13. Exapmle 3. Time evolution of power (top) and $H R$ (bottom)

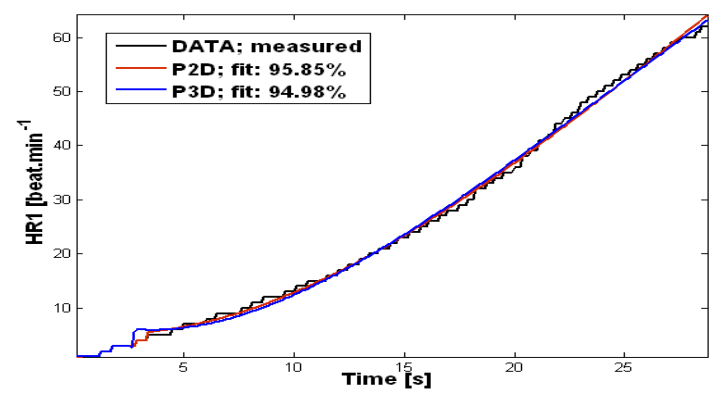

Figure 14. Exapmle 3. Results of different dynamical models used for estimation of WAT equations

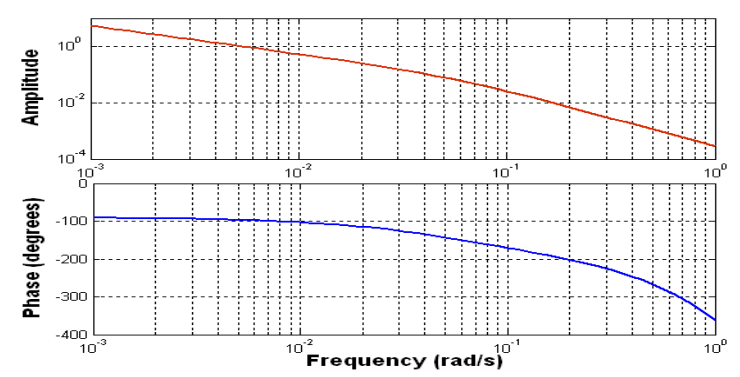

Figure 15. Exapmle 2. Bode diagram (P2D only)

\section{Discussion}

The results of estimation of the open-loop dynamics of physiological parameters based on laboratory exercise WAT tests and using linear models for 3 different subjects was presented. Despite this variability in the subject, also the model for oxygen uptake and cardiac output can be used for approximation and comparison of different physical activities. It is important to note, that estimated results are applicable only when load range and range of $H R$ measured in laboratory test and range of $H R$ for other physical activities are similar. There are some more practical applications: using given data energy expenditure during physical activity could be accurately calculated, and dietary recommendations could be exactly based on precisely obtained data.

\section{CONCLUSION}

Our study indicates that for exercise testing in laboratory, the linear dynamic model can be used for subject parameters estimation. This approach can be used for set of load and also cardiovascular parameters for different physical activities when only heart rate is measured.

\section{REFERENCES}

[1] O. Bar-Or, "The Wingate anaerobic test: an update on methodology, reliability and validity," Sports Medicine 4:1987, pp. 381-394.

[2] O. Bar-Or, R. Dotan, O. Inbar, "A $30 \mathrm{~s}$ all-out ergometric test: its reliability and validity for anaerobic capacity," Isr J Med Sci 13:126, 1977.

[3] J. A. Evans, H. A. Quinney, "Determination of resistance settings for anaerobic power testing, " Canadian Journal of Applied Sport Sciences. 6. 1981, pp. 53-56.

[4] D.Vandewalle, P. Gilbert, H. Monod, (1987). "Standard anaerobic tests," Sports Medicine. 4, 1987, pp. 268-289.

[5] M. Tossavainen, A. Nummela, L. Paavolainen, A. Mero, H. Rusko, "Comparison of two maximal anaerobic cycling tests," International Journal of Sports Medicine. 17 (S 2), 1996, pp. S120-S124.

[6] A. Nummela, M. Alberts, R. P. Rjintjes, P. Luhtanen, H. Rusko, "Reliability and validity of the maximal anaerobic running test," International Journal of Sports Medicine. 17 (S 2), 1996, pp. S97-S102.

[7] T. J. Hazell, R. MacPherson, B. Gravelle, P. Lemon, "10 or 30-s sprint interval training bouts enhance both aerobic and anaerobic performance," European Journal of Applied Physiology. 110, 2010, pp. 153-160.

[8] L. Franklin, L. "Accurate assessment of work done and power during a Wingate anaerobic test," Appl. Physiol. Nutr. Metab. 32, 2007, pp. 225-23.

[9] J. D. Coso, R. Mora-Rodriguez, "Validity of cycling peak power as measured by a short-sprint test versus the Wingate anaerobic test," Applied Physiology, Nutrition, and Metabolism. 31, 2006, pp.186-189.

[10] R. Lericollais, A. Gauthier, N. Bessot, D. Davenne, "Diurnal evolution of cycling biomechanical parameters during a 60-s Wingate test," Scandinavian Journal of Medicine and Science in Sports. 21, 2010, pp. 1-9.

[11] E. Bai, "Decoupling the linear and nonlinear parts in Hammerstein model identification," Automatica, vol. 40, 2004, pp. 671-676.

[12] I. Goethals, K. Pelckmans, J. Suykens, and B. D. Moor. "Identification of mimo Hammerstein models using least squares support vector machines," Automatica, vol. 41, 2005, pp. 1263-1272.

[13] S. Su, L. Wang, B. Celler, and A. Savkin, "Estimation of oxygen consumption for moderate exercises by using a Hammerstein model," Proc. 28th Annual Int. Conf. IEEE Engineering in Medicine and Biology Society (EMBS), New York, Sep. 2006, pp. 3427-3430. 